\title{
Doping effects on the Raman spectra of silicon nanowires
}

\author{
Chao-Yu Meng, Jui-Lin Chen, and Si-Chen Lee \\ Graduate Institute of Electronics Engineering and Department of Electrical Engineering, National Taiwan University, Taipei, 106, \\ Taiwan
}

Chih-Ta Chia

Department of Physics, National Taiwan Normal University, Taipei, 100, Taiwan

(Received 21 October 2005; revised manuscript received 31 March 2006; published 9 June 2006)

\begin{abstract}
Un-doped, $N$-type, and $P$-type doped silicon nanowires (SiNWs) were grown at $460{ }^{\circ} \mathrm{C}$ and 25 Torr via the vapor-liquid-solid (VLS) mechanism. The intensity ratio of anti-Stokes/Stokes $\left(I_{\mathrm{AS}} / I_{\mathrm{S}}\right)$ peaks is used as an index of the sample temperature. Different SiNWs exhibit different Raman frequency shifts because their compressive stresses due to heating differ. The slopes of the $I_{\mathrm{AS}} / I_{\mathrm{S}}$ peak ratio versus the Raman frequency for boron-doped, un-doped, phosphorous-doped SiNWs, and bulk $\mathrm{Si}$ are $-0.078,-0.036,-0.035$ and -0.02 per $\mathrm{cm}^{-1}$, respectively. The different slopes reveal the different heating-induced compressive stresses in the SiNWs with different dopants and bulk Si.
\end{abstract}

DOI: 10.1103/PhysRevB.73.245309

PACS number(s): 78.67.Lt, 78.30.Am

\section{INTRODUCTION}

Nanomaterials have attracted increasing attention in recent years owing to their quantum size effects and potential technological applications. Silicon nanowire (SiNW) is one of the most attractive materials because currently available semiconductor fabrication technologies are based mainly on Si. Accordingly, investigating the characteristics of SiNWs and developing nanowires technology are important. Raman spectroscopy is employed to investigate the quantum confinement effect, because it is nondestructive. Various works on the Raman spectra of undoped SiNWs have been published in the last few years. ${ }^{1-6}$ The effects of heating on the sample caused by laser irradiation are believed possibly to be the main reason for the downshift and broadening of Raman spectra of SiNWs. ${ }^{7}$ The Fano effects should also be taken into account to characterize the asymmetric line shape. ${ }^{6}$ The compressive stress is also included to plot the intensity ratio of anti-Stokes/Stokes $\left(I_{\mathrm{AS}} / I_{\mathrm{S}}\right)$ ratio versus the Raman peak downshift. Previous studies have dealt only with un-doped SiNWs. In this work, the doping of SiNWs is studied and an index employed to clarify the effect of the compressive stress on the Raman peak shift in different samples.

\section{EXPERIMENTS}

Corning $1737 \mathrm{~F}$ glass was used as the template herein to avoid confusion from Raman spectra between the silicon substrate and the SiNWs. $20 \mathrm{~nm}$ Au colloids were sprayed onto the glass surface as the catalyst during vapor-liquidsolid (VLS) growth. For comparison, un-doped, borondoped, and phosphorus-doped SiNWs were grown by lowpressure chemical vapor deposition (LPCVD) to obtain the Raman spectra. The flow ratios of $\mathrm{B}_{2} \mathrm{H}_{6}$ and $\mathrm{PH}_{3}$ to $\mathrm{SiH}_{4}$ are 100 and $700 \mathrm{ppm}$, respectively. The process temperature and pressure were maintained at $460{ }^{\circ} \mathrm{C}$ and 25 Torr during the depositions. Raman spectra were obtained using a Jobin Yvon T64000 system and a coherent Verdi-V10 $532 \mathrm{~nm}$ laser. A $50 \times$ microscopy objective lens was utilized in this experiment.

\section{RESULTS AND DISCUSSION}

Four possible causes of the downshift of the Raman peak are considered to explain its relationship with $I_{\mathrm{AS}} / I_{\mathrm{S}}$. These are (i) the rise of temperature, (ii) the high carrier densities, (iii) the quantum confinement effect, and (iv) the stress in the SiNWs. First, most of the SiNWs in the thin film are not in contact with the substrate but are suspended by other nanowires, causing poor thermal contact with the substrate. Therefore, the heating effect of incident laser on SiNWs exceeds that on the silicon bulk substrate and causes a downshift in the Raman peaks and increases the intensity ratio of the anti-Stokes/Stokes $\left(I_{\mathrm{AS}} / I_{\mathrm{S}}\right)$ peaks. Second, given sufficiently high doping levels, electrons or holes participate in the light-scattering process and excite the free carriers to the next higher conduction band or the valance band. The combined effect of the electronic continuum and the LO (TO) phonons results in the asymmetric Fano line shape and the downshift of the Raman peak. Third, as the diameter of the SiNW shrinks, the selection rule is relaxed and the Raman peak is downshifted. Fourth, the stress in the SiNW changes the lattice constant, affecting the position of the Raman peak. The total Raman peak shift is the sum of the four effects described above.

Figures 1(a)-1(c) display SEM images of undoped, boron-doped and phosphorous-doped SiNWs, respectively. The mean diameters calculated from the SEM images are 24.7, 27.8, and $26.2 \mathrm{~nm}$. Figure 2 presents the transmission spectra of undoped, boron-doped and phosphorous-doped SiNWs. They are not normalized to the density of the SiNWs. Kasuya et al. ${ }^{8}$ investigated one-dimensional (1D) carbon nanotubes and found that the dip of the transmission spectrum corresponds to resonance. However, the transmission spectrum at the laser wavelength $(532 \mathrm{~nm})$ shown in Fig. 2 is very smooth, therefore, the resonance effects near the measurement wavelength can be ignored. Thus, samples with the same $I_{\mathrm{AS}} / I_{\mathrm{S}}$ ratio can be treated as being at the same temperature.

Figure 3(a) shows the Raman spectra of undoped SiNWs, showing the anti-Stokes and Stokes peaks. The correspond- 


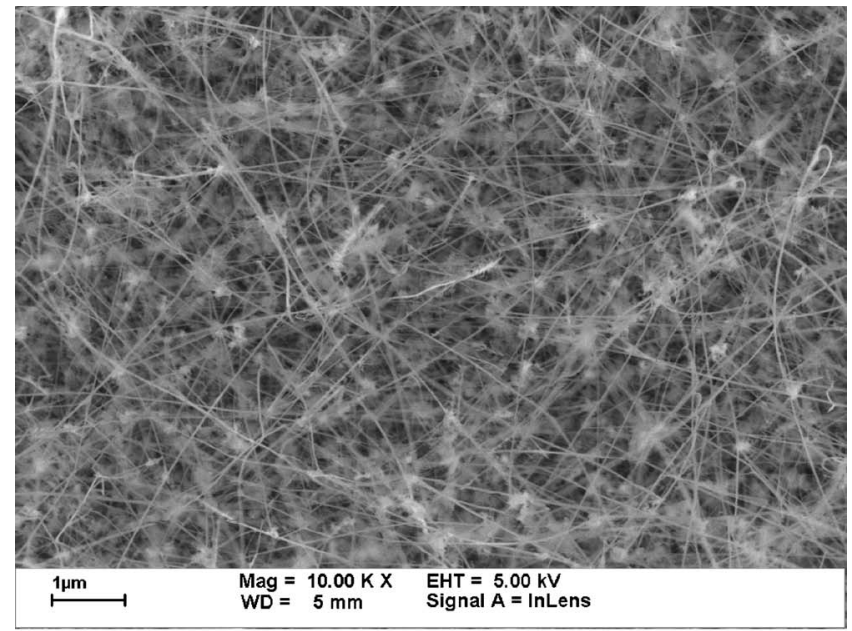

(a)

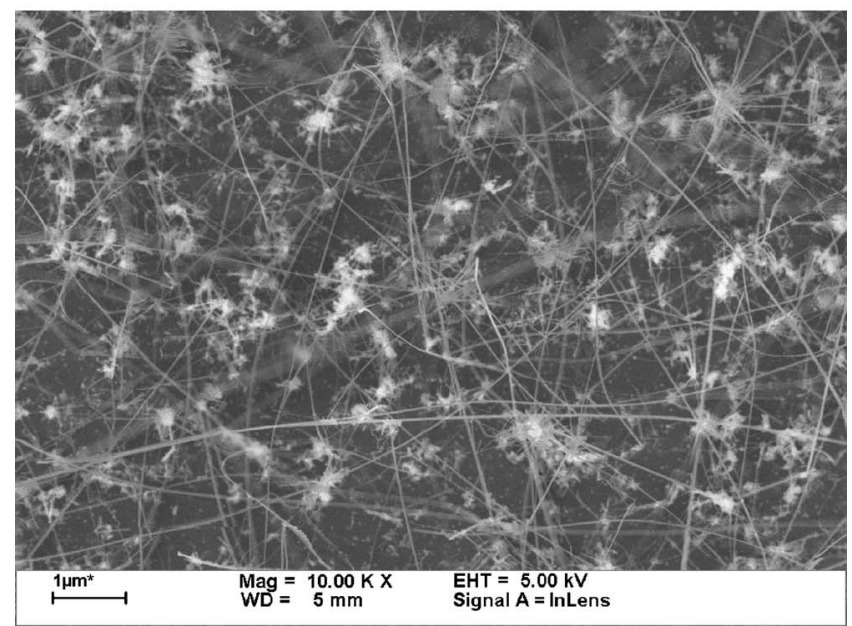

(b)

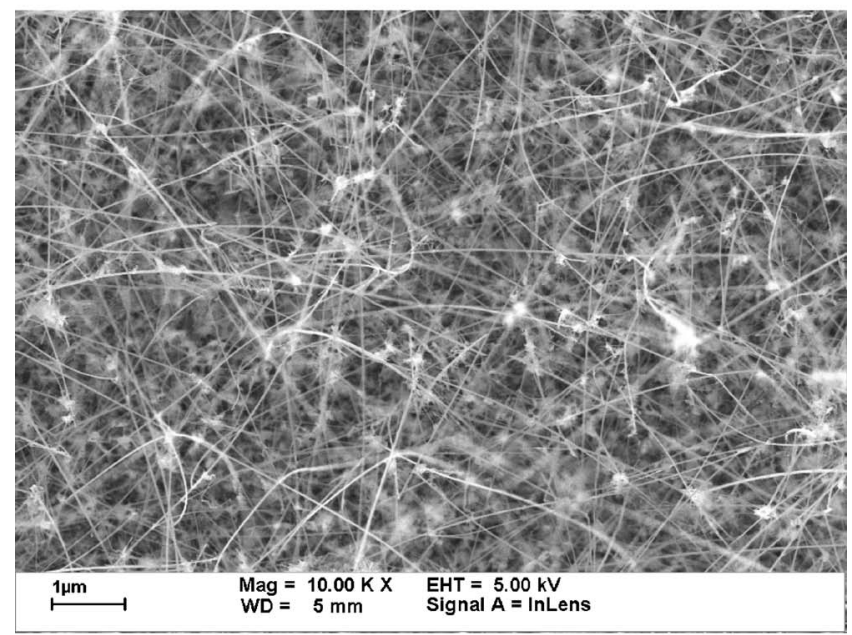

(c)

FIG. 1. The SEM image of (a) un-doped, (b) boron-doped, and (c) phosphorous-doped SiNWs.

ing temperature calculated from the $I_{\mathrm{AS}} / I_{\mathrm{S}}$ ratio, based on the assumption of a classical Bose population, is $21^{\circ} \mathrm{C}$. Figure 3(b) plots the $I_{\mathrm{AS}} / I_{\mathrm{S}}$ ratio of silicon LO (TO) phonons of undoped, boron-doped, and phosphorous-doped SiNWs

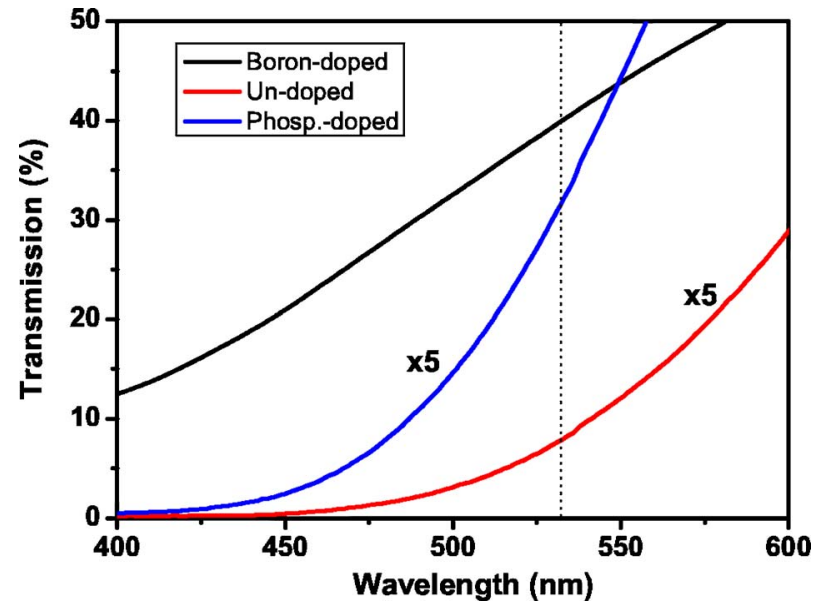

FIG. 2. (Color online) The transmission spectra of undoped, boron-doped, and phosphorous-doped SiNWs near the laser wavelength of $532 \mathrm{~nm}$. The absolute transmissions are not normalized to the densities of the SiNWs.

grown at $460{ }^{\circ} \mathrm{C}$ versus laser power density. The $I_{\mathrm{AS}} / I_{\mathrm{S}}$ ratios of undoped and boron-doped SiNWs behave almost the same as the laser power density increases, whereas the $I_{\mathrm{AS}} / I_{\mathrm{S}}$ ratio of the phosphorous-doped SiNWs is higher, and increases more quickly as a function of the laser power density, perhaps because of the electron doping of the SiNWs.

Figures 4(a)-4(c) present the Stokes Raman spectra of undoped, boron-doped, and phosphorous-doped SiNWs, respectively. The laser power density is kept the same for the three samples. As the laser power density increases, the $520 \mathrm{~cm}^{-1}$ Raman peak downshifts and asymmetrically broadens. Comparing Figs. 4(a)-4(c) reveals that the full widths at half maximums (FWHMs) of the undoped and phosphorous-doped SiNWs, 6.6 and $8.8 \mathrm{~cm}^{-1}$, at an incident laser power density of $8.4 \times 10^{4} \mathrm{~W} / \mathrm{cm}^{2}$, are broadened more than those of boron-doped SiNWs, $4.5 \mathrm{~cm}^{-1}$. Hence, the phonon lifetime in undoped and phosphorous-doped SiNWs is shorter than that in boron-doped SiNWs. The phonon lifetime is determined by the anharmonic interactions of the optical phonons, which decay into combinations of phonons of lower energy, indicating that the optical phonon lifetime of phosphorous-doped SiNWs is approximately 33\% shorter than that of the undoped SiNWs and 50\% shorter than that of boron-doped SiNWs. Besides, the $495 \mathrm{~cm}^{-1}$ peak may be associated with the strained $\mathrm{Si}$ after LPCVD growth.

The solid lines in Figs. 4(a)-4(c) represent the fits of the Raman spectra to a Fano resonance line shape ${ }^{9}$

$$
I(\omega)=A \frac{(q+\varepsilon)^{2}}{\left(1+\varepsilon^{2}\right)}, \quad \varepsilon=\frac{\left(\omega-\omega_{o}\right)}{\Gamma},
$$

where $\omega_{o}$ is the renormalized phonon frequency in the presence of coupled scattering; $q$ is the asymmetry parameter ( $q \rightarrow \infty$ gives a Lorentzian), and $\Gamma$ is the linewidth parameter. Fano interference scattering in doped bulk silicon has been extensively dealt with in earlier Raman studies. ${ }^{10-14}$ The equation is applied to fit the experimental results to elucidate the characteristics of doping and photon-induced carriers in the SiNWs. Figures 5(a)-5(c) plot Fano parameters extracted 

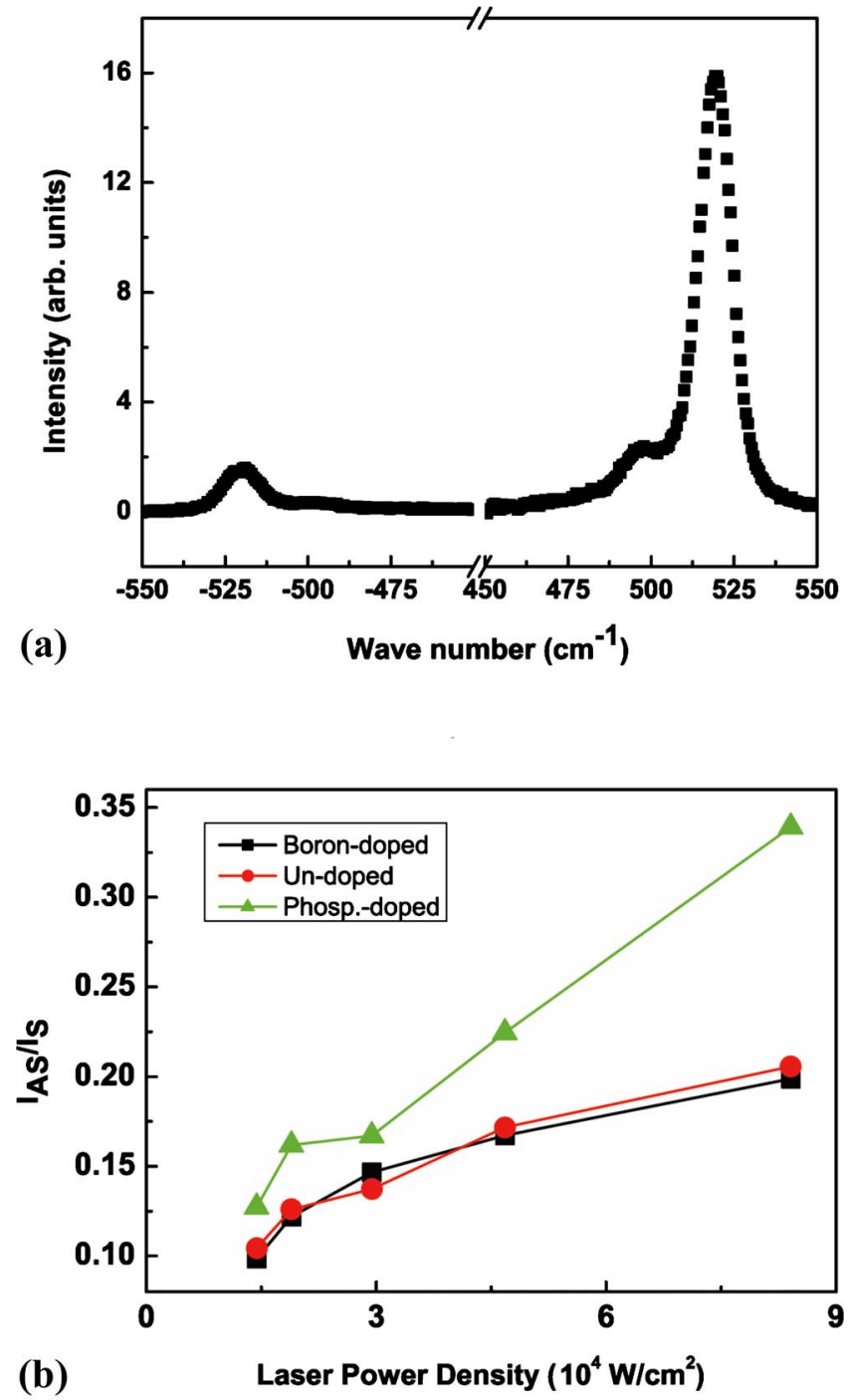

FIG. 3. (Color online) (a) The Raman spectra showing the antiStokes and Stokes peaks of undoped SiNWs. (b) The intensity ratio of anti-Stokes/Stokes $\left(I_{\mathrm{AS}} / I_{S}\right)$ peaks as a function of laser power density for undoped, boron-doped, and phosphorous-doped SiNWs.

from the fitting curves in Fig. 4 versus incident laser power density. Comparing Fig. 5(a) with Fig. 3(b) demonstrates that when the incident laser power density is below 1.4 $\times 10^{4} \mathrm{~W} / \mathrm{cm}^{2}$, the Raman peaks of undoped and borondoped samples are around $520 \mathrm{~cm}^{-1}$, suggesting that the samples are at room temperature and the laser heating effect has not yet occurred. However, for the phosphorous-doped samples, the Raman downshift is clear and the heating effect must start at this moment. As the laser power density increases, the downshifts of all three samples increase because of the heating effect. Notably, the downshift of the phosphorous-doped SiNWs changes the most, followed by that of the undoped SiNWs and that of the boron-doped, in that order, because the heavy doping of SiNWs by phosphorous may reduce the band gap of the SiNW, increasing the total power absorbed by the phosphorous-doped SiNWs and further increasing the temperature. The downshift of the undoped SiNWs increases more quickly than that of the boron-
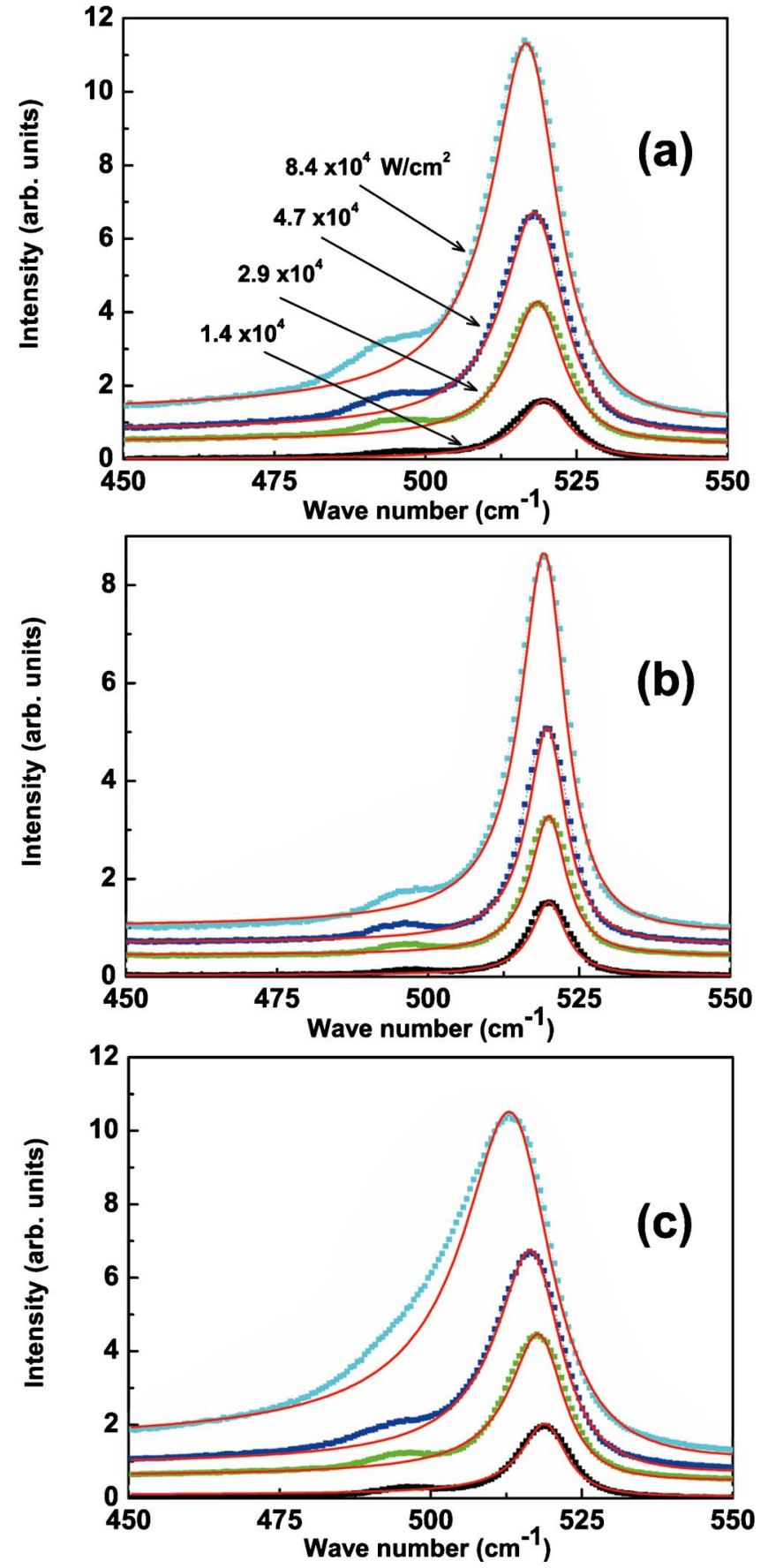

FIG. 4. (Color online) Stokes Raman spectra of (a) undoped, (b) boron-doped, and (c) phosphorous-doped SiNWs vs incident laser power density. The solid lines represent the fits of data using Fano resonance line shape.

doped SiNWs perhaps because the heat-induced stress of boron-doped SiNWs is larger, as will be discussed later. These results also correspond to the variation of the $1 / q$ factor, as shown in Fig. 5(c), which is proportional to the free carrier concentration. ${ }^{10,11}$ Furthermore, when the laser power density was below $1.4 \times 10^{4} \mathrm{~W} / \mathrm{cm}^{2}$ and the samples were at room temperature, the Raman peak downshift was zero, indicating that the quantum confinement effects could be neglected. The results also agree with those obtained in a simulation by Piscanec et al. ${ }^{7}$ 


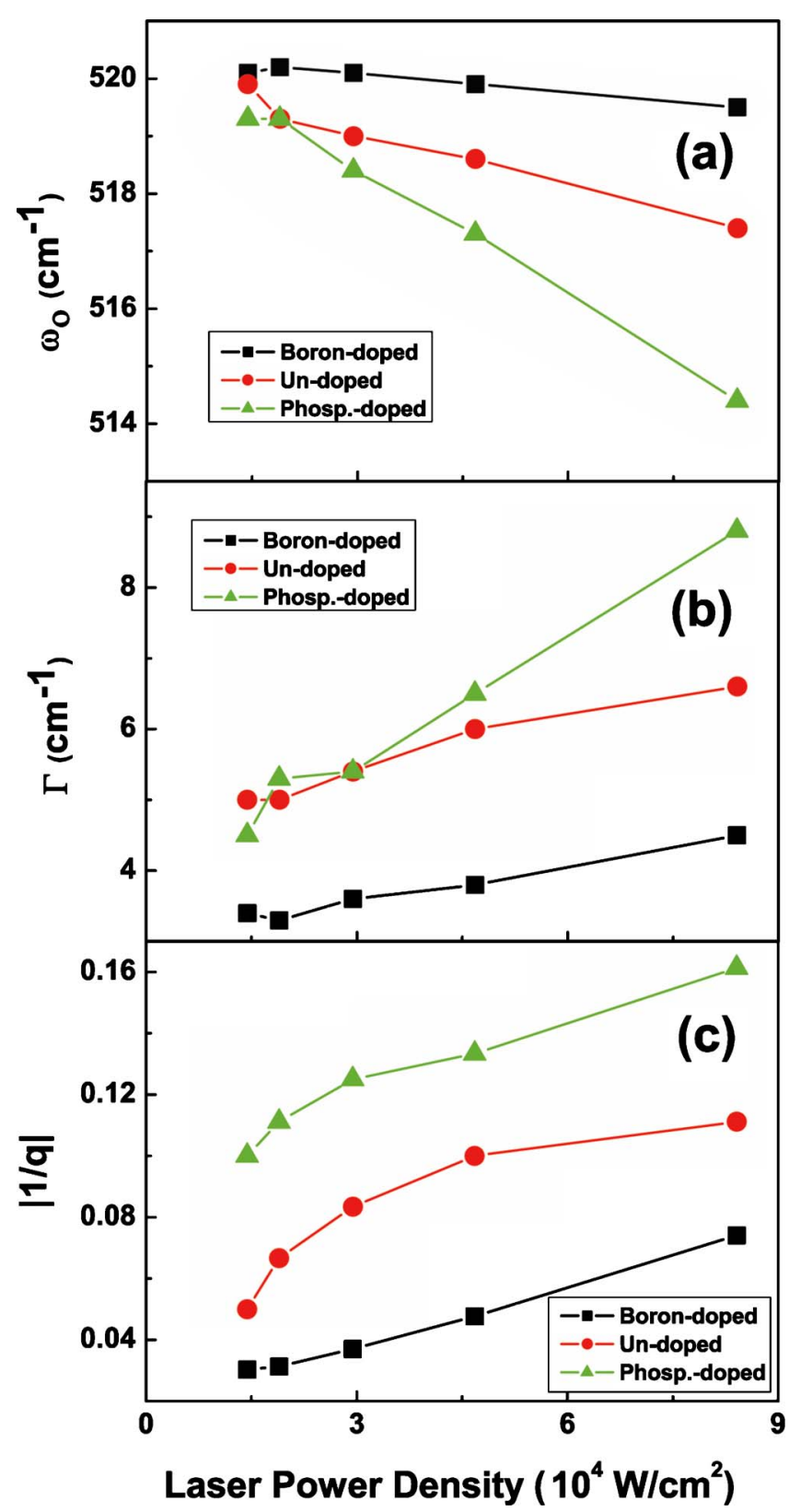

FIG. 5. (Color online) Parameters extracted by fitting the Fano resonance line shape to Fig. 4, (a) Raman peak frequency $\omega_{o}$, (b) linewidth $\Gamma$, and (c) asymmetry factor $|1 / q|$.

A simple absorption model of cylindrical SiNW, which is schematically depicted in Fig. 6, is proposed here to calculate the total absorbed energy, and thus quantify the effects of the injection of free carriers,

$$
\begin{aligned}
E= & 2 \times P \times l \int T\left(1-e^{-2 \alpha \gamma \cos \varphi}\right) \gamma \sin \left(\frac{\pi}{2}-\varphi\right) \\
& \times \frac{n_{s} \cos \varphi}{\sqrt{1-n_{s}^{2} \sin ^{2} \varphi}} d \varphi,
\end{aligned}
$$

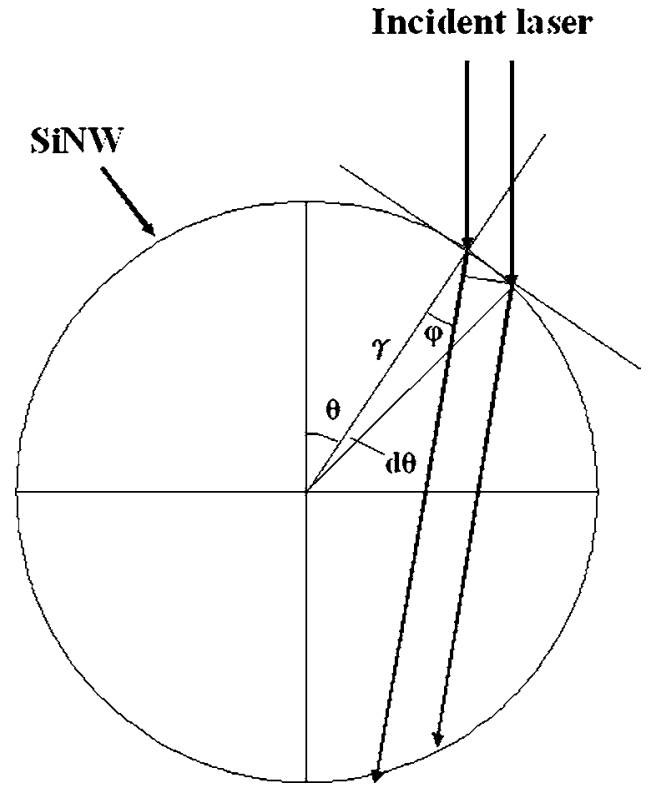

FIG. 6. Model showing the absorption of incident laser power by cylindrical shape SiNW. The arrows represent incident and refractive light path in the silicon nanowires.

$$
\begin{aligned}
T= & \frac{4}{n_{s} \cos \varphi \sqrt{1-n_{s}^{2} \sin ^{2} \varphi}} / \\
& \times\left(\frac{1}{n_{s} \cos \varphi}+\frac{1}{\sqrt{1-n_{s}^{2} \sin ^{2} \varphi}}\right)^{2},
\end{aligned}
$$

where $T$ denotes the transmittance of the incident light in the SiNWs, $P$ represents the incident laser power density, $l$ is the length of the SiNWs covered by the laser spot, $\alpha$ is the absorption coefficient, and $n_{s}$ is the refractive index of the SiNWs. Therefore, the free carrier generation rate per unit volume can be described as

$$
G=\frac{E}{l \pi \gamma^{2} e_{\text {photon }}} .
$$

Combining Eq. (2) with Eqs. (3) and (4) yields the free carrier generation rate per unit volume directly. Substituting into the parameters described above $\gamma=10 \mathrm{~nm}, \alpha=10^{4} \mathrm{~cm}^{-1}$ for undoped SiNWs, and $P=8.4 \times 10^{4} \mathrm{~W} / \mathrm{cm}^{2}$ yields a free carrier generation rate per unit volume of $3.4 \times 10^{27} \mathrm{~cm}^{-3}$. Since the injected free carrier generation rate is high and the SiNW has a small diameter and very large length, the free carrier concentrations can be concisely derived as

$$
G=C_{a} n^{3},
$$

where $C_{a}$ is the Augar coefficient, and $G$ is the free carrier generation rate per unit volume. The low injection recombination term and the radiative recombination term are ignored for the high injection rate; the diffusion term can also be reasonably ignored because the diameter of SiNWs is small. At such a high injection rate, $C_{a}$ can be reasonably chosen as $3.8 \times 10^{-31} \cdot{ }^{15,16}$ Hence, the corresponding free carrier densities can be determined from the above equations to be 2 


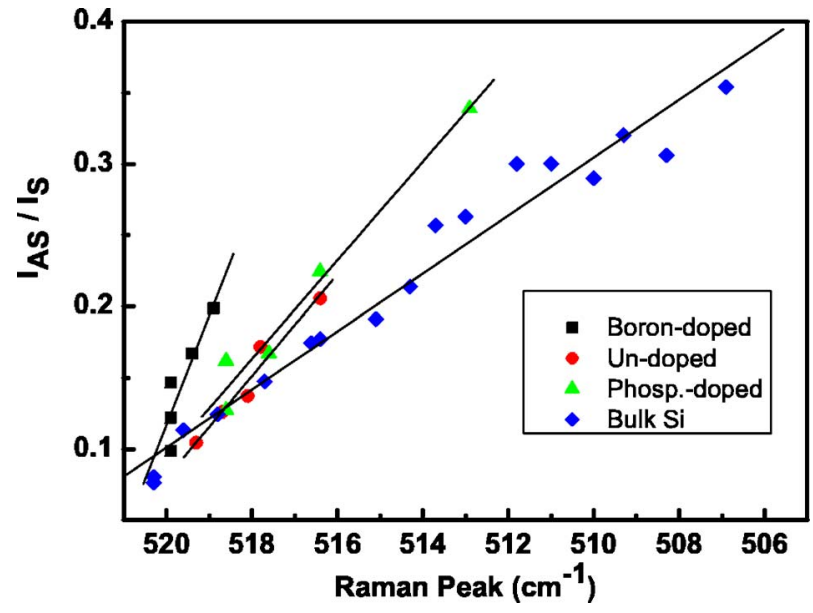

FIG. 7. (Color online) The $I_{\mathrm{AS}} / I_{S}$ ratio vs Raman peak shift for undoped, boron-doped, phosphorus-doped SiNWs and bulk silicon.

$\times 10^{19} \mathrm{~cm}^{-3}$, which result is also consistent with the assumption made above.

Comparing the shift in frequency of the undoped SiNWs with that of the $n$-type doped bulk silicon ${ }^{12}$ reveals that for a Raman shift of $2 \mathrm{~cm}^{-1}$, the doping concentration in bulk silicon must be about $1.5 \times 10^{20} \mathrm{~cm}^{-3}$; however, the calculated concentration was only $2 \times 10^{19} \mathrm{~cm}^{-3}$, which is an order of magnitude lower. Besides, if the doping gas, $\mathrm{B}_{2} \mathrm{H}_{6}$ and $\mathrm{PH}_{3}$, can be $100 \%$ dissociated and dissolved in the $\mathrm{Au} / \mathrm{Si}$ alloy to form doped SiNWs in a manner governed by the gas phase concentration ratio, then the boron and phosphorous concentrations can be estimated to be $1 \times 10^{19}$ and $3.5 \times 10^{19} \mathrm{~cm}^{-3}$, respectively, which are still far below the values needed, suggesting that the downshift in the Raman peak depends mainly on the effect of heating rather than on the concentration of free carriers.

Figure 7 plots the $I_{\mathrm{AS}} / I_{\mathrm{S}}$ ratio against the Raman peak shift for undoped, boron-doped, phosphorous-doped SiNWs, and bulk silicon. The bulk Si curve represents the reference curve at different heating temperatures. The solid lines represent linear fits to the experimental data. The slopes $S$ of the linear fit to the curve of $I_{\mathrm{AS}} / I_{\mathrm{S}}$ ratio versus the Raman peak plot for boron-doped, undoped, phosphorous-doped SiNWs and bulk $\mathrm{Si}$ are $-0.078,-0.036,-0.035$, and -0.02 per $\mathrm{cm}^{-1}$, respectively. The fitting results demonstrate that the slopes of the undoped and phosphorous-doped SiNWs are very close to each other but the slope of the boron-doped SiNWs significantly exceeds the others. The slopes of the SiNWs all exceed that of bulk silicon. Since the effects of the carrier concentration and quantum confinement on the Raman peak position can be neglected, the downshift of the Raman peak is due mainly to the heating effect of SiNWs, and should be related to the heating behavior of the bulk silicon. However, the downshifts of the Raman peak of the SiNWs at a given $I_{\mathrm{AS}} / I_{\mathrm{S}}$ ratio are all smaller than those of bulk silicon, revealing the existence of an additional factor in suppressing the downshift of the Raman peak because of the heating-induced compressive stress in the SiNW. Boron-doped SiNWs exhibit the most strongly temperature-dependent compressive stress.

Comparing Fig. 7 with Fig. 5(a) clearly shows that even when the Raman peaks of the undoped and phosphorousdoped SiNWs are differently downshifted when plotted against incident laser power density, they have almost the same $I_{\mathrm{AS}} / I_{\mathrm{S}}$ ratios and similar slopes $S$ at the same Raman peak frequency, revealing that the compressive stresses for these two samples are the same when they are at the same temperature (same $I_{\mathrm{AS}} / I_{\mathrm{S}}$ ratio). However, the boron-doped samples exhibit different compressive stress behaviors because they have different Raman frequencies and slopes, as described above. The slopes of undoped and phosphorousdoped SiNWs are shallower than that of boron-doped SiNWs because the compressive stress caused by heating is less dependent on temperature. Therefore, when three types of SiNW are grown at the same temperature as in this experiment, the boron-doped SiNWs may be of better quality but have greater compressive stress, and the phosphorous-doped SiNWs start to heat up earlier, because the absorption coefficient is higher, increasing the temperature at a particular laser power irradiation.

\section{CONCLUSIONS}

In summary, suspended SiNWs are more easily heated by an incident laser than bulk silicon because they are in poor thermal contact with the substrate. Accordingly, the Raman line is downshifted and broadened because of the heating effect and the associated compressive stress. The quantum confinement and carrier effects do not dominate the downshift in the Raman peak. However, varying the doping level changes the absorption coefficient of the SiNWs, and thus temperature. Finally, the slope of $I_{\mathrm{AS}} / I_{\mathrm{S}}$ ratio versus the Raman peak shift is proposed as an index of the compressive stress in various SiNWs.

\section{ACKNOWLEDGMENT}

The authors would like to thank the National Science Council of the Republic of China, Taiwan, for financially supporting this research under Contract No. NSC 93-2215E-002-046.
${ }^{1}$ B. Li, D. Yu, and S. L. Zhang, Phys. Rev. B 59, 1645 (1999).

${ }^{2}$ R. P. Wang, G. W. Zhou, Y. L. Liu, S. H. Pan, H. Z. Zhang, D. P. Yu, and Z. Zhang, Phys. Rev. B 61, 16827 (2000).

${ }^{3}$ S. L. Zhang, W. Ding Y. Yan, J. Qu, B. Li, L. Y. Li, K. T. Yue, and D. Yu, Appl. Phys. Lett. 81, 4446 (2002).
${ }^{4}$ D. P. Yu, Z. G. Bai, Y. Ding, Q. L. Hang, H. Z. Zhang, J. J. Wang, Y. H. Zou, W. Qian, G. C. Xiong, H. T. Zhou, and S. Q. Feng, Appl. Phys. Lett. 72, 3458 (1998).

${ }^{5}$ S. Hofmann, C. Ducati, R. J. Neill, S. Piscanec, A. C. Ferrari, J. Geng, R. E. Dunin-Borkowski, and J. Robertson, J. Appl. Phys. 
94, 6005 (2003).

${ }^{6}$ R. Gupta, Q. Xiong, C. K. Adu, U. J. Kim, and P. C. Eklund, Nano Lett. 3, 627 (2003).

${ }^{7}$ S. Piscanec, M. Cantoro, A. C. Ferrari, J. A. Zapien, Y. Lifshitz, S. T. Lee, S. Hofmann, and J. Robertson, Phys. Rev. B 68, 241312(R) (2003).

${ }^{8}$ A. Kasuya, M. Sugano, T. Maeda, Y. Saito, K. Tohji, H. Takahashi, Y. Sasaki, M. Fukushima, Y. Nishina, and C. Horie, Phys. Rev. B 57, 4999 (1998).

${ }^{9}$ U. Fano, Phys. Rev. 124, 1866 (1961).

${ }^{10}$ M. Balkanski, K. P. Jain, R. Beserman, and M. Jouanne, Phys.
Rev. B 12, 4328 (1975).

${ }^{11}$ F. Cerdeira and M. Cardona, Phys. Rev. B 5, 1440 (1972).

${ }^{12}$ M. Chandrasekhar, J. B. Renucci, and M. Cardona, Phys. Rev. B 17, 1623 (1978).

${ }^{13}$ N. H. Nickel, P. Lengsfeld, and I. Sieber, Phys. Rev. B 61, 15558 (2000).

${ }^{14}$ R. Beserman and T. Bernstein, J. Appl. Phys. 48, 1548 (1977).

${ }^{15}$ V. Magidson and R. Beserman, Phys. Rev. B 66, 195206 (2002).

${ }^{16}$ P. Jonsson, H. Bleichner, M. Isberg, and E. Nordlander, J. Appl. Phys. 81, 2256 (1997). 